\title{
Lista actualizada de quirópteros de los Departamentos de Loreto, Ucayali y Madre de Dios (Perú)
}

\author{
Gilberto Josimar Fernández-Arellano ${ }^{1,2^{*}}$, María Isabel Torres-VÁsqueZ ${ }^{2}$, \\ ${ }^{1}$ Instituto Nacional de Pesquisas da Amazônia, \\ ${ }^{2}$ Universidad Nacional de la Amazonia Peruana. \\ *Correo electrónico del autor: giliusfernandez1@gmail.com
}

DOI: http://dx.doi.org/10.14709/BarbJ.6.1.2013.10

English title: An updated list of bats from the Departments of Loreto, Ucayali and Madre de Dios (Peru)

\begin{abstract}
The present review reports a total of 127 bats species, grouped in 57 genera and 8 families. We highlight the first cites of 5 species for Loreto Department (Artibeus bogotensis, Artibeus phaeotis, Artibeus watsoni, Eumops delticus, Peropteryx pallidoptera), and just 1 species for Loreto and Madre de Dios Departments (Vampyressa pusilla). These 6 species represent new records for Peru. According to the baseline (Gardner 2007), also extends the distribution range of another 18 species. The total number of species recorded is distributed among the families Phyllostomidae (63,8\%), Molossidae $(13,4 \%)$, Emballonuridae (8.7\%), Vespertilionidae (8.7\%), Thyropteridae (2,4\%), Noctilionidae (1,6\%), Furipteridae $(0,7 \%)$, and Mormoopidae $(0,7 \%)$. Vampyressa melissa is a vulnerable species according to IUCN. We also report an increase in published articles and conducted undergraduate thesis related to bats.
\end{abstract}

Keywords: Amazon, bats, conservation, inventory, IUCN.

Resumen: La presente revisión reporta un total de 127 especies de quirópteros, agrupadas en 57 géneros y 8 familias. Se resaltan las primeras citas de 5 especies para el Departamento de Loreto (Artibeus bogotensis, Artibeus phaeotis, Artibeus watsoni, Eumops delticus, Peropteryx pallidoptera), y solo 1 especie para los Departamentos de Loreto y Madre de Dios (Vampyressa pusilla). Estas 6 especies representan nuevos registros para el Perú. Según la referencia básica (Gardner 2007), también se extiende el rango de distribución de otras 18 especies. El total de especies registradas se distribuye entre las familias Phyllostomidae (63,8\%), Molossidae (13,4\%), Emballonuridae (8,7\%), Vespertilionidae (8,7\%), Thyropteridae (2,4\%), Noctilionidae (1,6\%), Furipteridae (0,7\%), y Mormoopidae (0,7\%). Vampyressa melissa es una especie vulnerable según la IUCN. También se reporta un incremento en la publicación de artículos y en la realización de tesis de pre-grado orientados hacia el grupo de los quirópteros.

Palabras clave: Amazonia, murciélagos, conservación, inventarios, IUCN.

\section{INTRODUCCIÓN}

Los quirópteros son el segundo orden más diverso de mamíferos a nivel mundial y el de mayor diversidad a nivel local (Sosa et al. 2008). Dentro de la región neotropical, el Perú es uno de los países con mayor diversidad de quirópteros (152 y 165, Pacheco 2002 y Pacheco et al. 2009, respectivamente), junto a Brasil (167 y 174, Reis et al. 2007 y Paglia et al. 2012, respectivamente), y Colombia (178, Alberico et al. 2000). Ecuador y Bolivia (143 y 103, Tirira 2007 y Salazar et al. 2002, respectivamente), también tienen una gran diversidad. La mayor diversidad de mamíferos del Perú se encuentra en el llano amazónico (Voss \& Emmons 1996, Pacheco 2002, Pacheco 2009), que incluye principalmente los Departamentos de Loreto, Ucayali, y Madre de Dios. 


\section{DEPARTAMENTOS DEL PERÚ

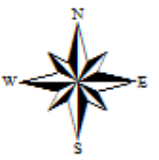

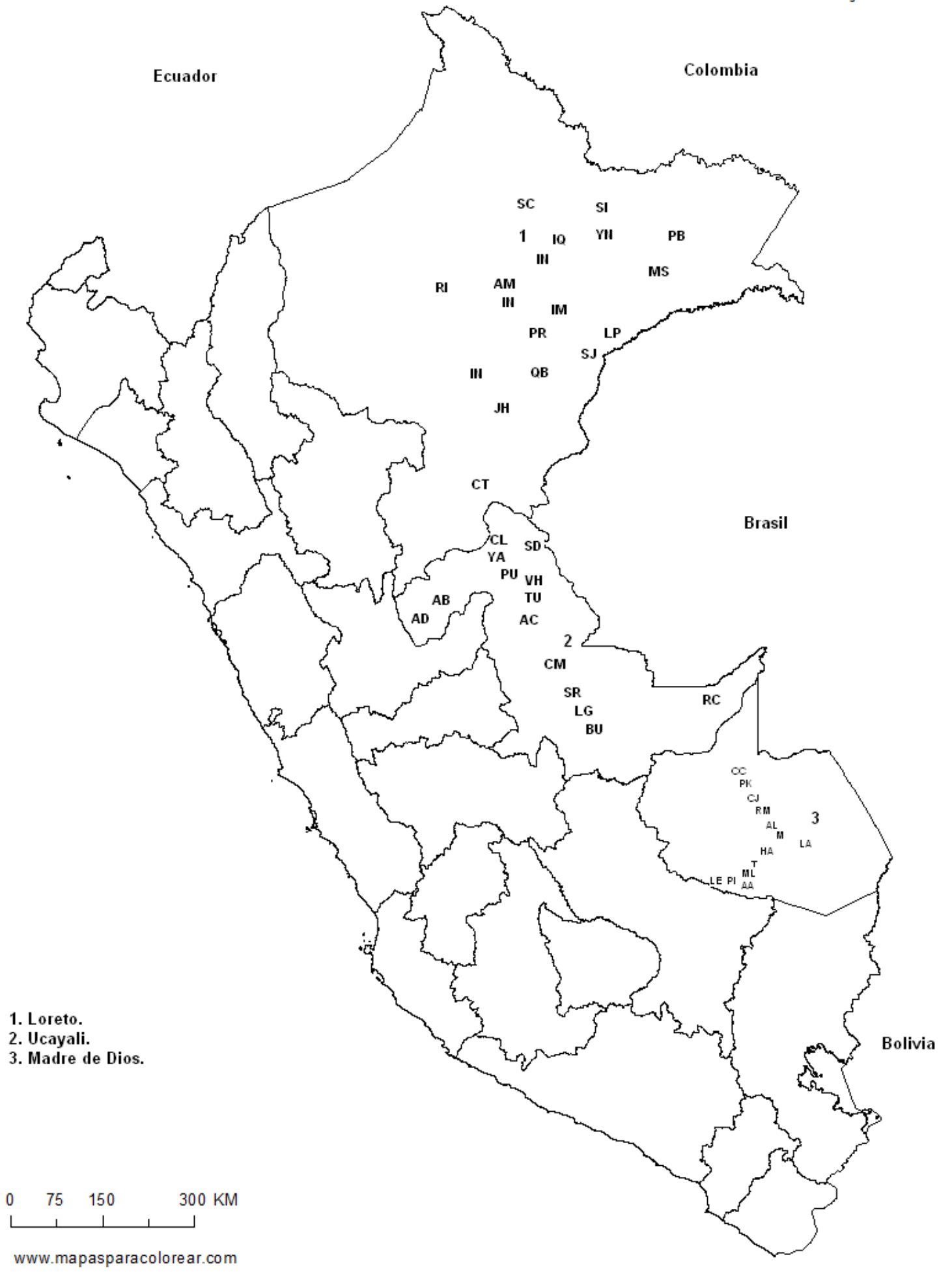

Fig. 1 Departamentos de Loreto, Ucayali y Madre de Dios con sus respectivos sitios de estudio.

Leyenda: todos los acrónimos por Departamento (Loreto, Ucayali y Madre de Dios) contenidos en el presente mapa son descritos detalladamente en la Tabla 1. Así mismo, todos los nuevos sitios de registro de quirópteros que actualizan a la referencia básica (Gardner 2007) se presentan detalladamente en la Figura 2. Por falta de espacio no se colocaron algunos acrónimos de los sitios de estudio en el Departamento de Madre de Dios (ver Tabla 1 y Figura 2). 
Debido a sus hábitos alimenticios los quirópteros desempeñan funciones ecológicas muy importantes para el mantenimiento de los ecosistemas naturales (Sosa et al. 2008). Ellos son componentes críticos para el desenvolvimiento de servicios ambientales tales como la dispersión de semillas (Medellín \& Gaona 1999, Wilson 2002), la polinización de flores, y la regulación del tamaño de las poblaciones de artrópodos y pequeños vertebrados (Wilson 2002). Sin embargo, estos servicios ambientales pueden verse afectados por la creciente transformación antropogénica del ambiente. La perturbación y destrucción del hábitat es la principal amenaza actual de las especies (Pacheco 2002). Al respecto, actividades económicas tales como la extracción de madera y petróleo así como la agricultura y ganadería favorecen la destrucción y modificación de hábitats críticos para la supervivencia de las especies en la Amazonia peruana (Escobedo 2003, Jorge \& Velazco 2006, Bravo \& Borman 2008, Díaz 2011).

El conocimiento sobre el estado de conservación de la fauna silvestre del Perú está disponible para el público en forma de listas oficiales de especies amenazadas, según los criterios de las instituciones involucradas (Ministerio de Agricultura) y la legislación nacional vigente en el respectivo año de su publicación. Al respecto, han sido publicadas dos resoluciones ministeriales; R.M. 01710-77-AG. (1977), R.M. 01082-90AG. (1990), y dos decretos supremos; D.S. 01399 AG. (1999), D.S. 034-2004 A.G. (2004), que proporcionan este tipo de información. Estas listas oficiales también pueden complementarse con información actualizada de organizaciones internacionales tales como la "International Union for the Conservation of Nature” (IUCN).

A pesar de los estudios realizados aún se conoce poco sobre la ecología, la distribución y el estado de conservación de los mamíferos ( $p$. ej. quirópteros) del Perú (Pacheco 2002) y en especial de aquellos distribuidos en la Amazonia peruana. El presente trabajo tiene por objetivo actualizar la lista de la referencia básica de Gardner (2007), de los quirópteros de los Departamentos de Loreto, Ucayali y Madre de Dios a partir de referencias bibliográficas, algunas poco accesibles y conocidas, y a través de la revisión online de diversas colecciones científicas.

\section{Materiales Y MÉtodos}

Varias fuentes de información fueron utilizadas para evaluar la literatura referida a los quirópteros. Se utilizó principalmente un motor de búsqueda online (Google Scholar) para identificar y acceder a estudios científicos relevantes y de interés para la presente revisión. Se utilizó una combinación de los siguientes términos claves para realizar una búsqueda más precisa: "mamíferos de Perú", "murciélagos de Perú", "chiropteros de Perú", "quirópteros de Perú", "amazon peruvian bats", "bats of Peru", "mammals of Peru", "peruvian bats". Se consultaron las listas de quirópteros capturados en artículos publicados en revistas indexadas y no indexadas, y trabajos de tesis de pre-grado (biblioteca de la facultad de Ciencias Biológicas de la Universidad Nacional de la Amazonia Peruana), realizados en los Departamentos de Loreto, Ucayali y Madre de Dios (Figura 1). Así mismo, se consultaron las bases de datos online de las colecciones de mamíferos de los principales museos de historia natural (American Museum of Natural History - AMNH, Field Museum of Natural History - FMNH, Lousiana State University Museum of Zoology - LSUMZ, Museum of Vertebrate Zoology - MVZ). También se consultó el gacetero online Global Biodiversity Information Facility - GBIF. Por último, se utilizó principalmente a Gardner (2007), como referencia básica para las distribuciones conocidas y para la posterior comparación con la nueva lista de especies.

\section{Resultados}

Nuevos registros, extensión de rango y estado de conservación

La presente revisión reporta un total de 127 especies de quirópteros, agrupadas en 57 géneros y 8 familias (Tabla 1). Se resaltan las primeras citas de 6 especies para el Departamento de Loreto, Artibeus watsoni (Saavedra 2010), Peropteryx pallidoptera (Lim et al. 2010), Artibeus phaeotis (Ramos 2011), Eumops delticus (Díaz 2011), Artibeus bogotensis (Calderón \& Pacheco 2012), y Vampyressa pusilla (López 2001, Bravo et al. 2008, Ramos 2011), esta última también nueva para el Departamento de Loreto y Madre de Dios. Estas 6 especies no habían sido registradas previamente para el Perú (Gardner 2007). Según esta referencia básica (Gardner 2007), también se extiende el 

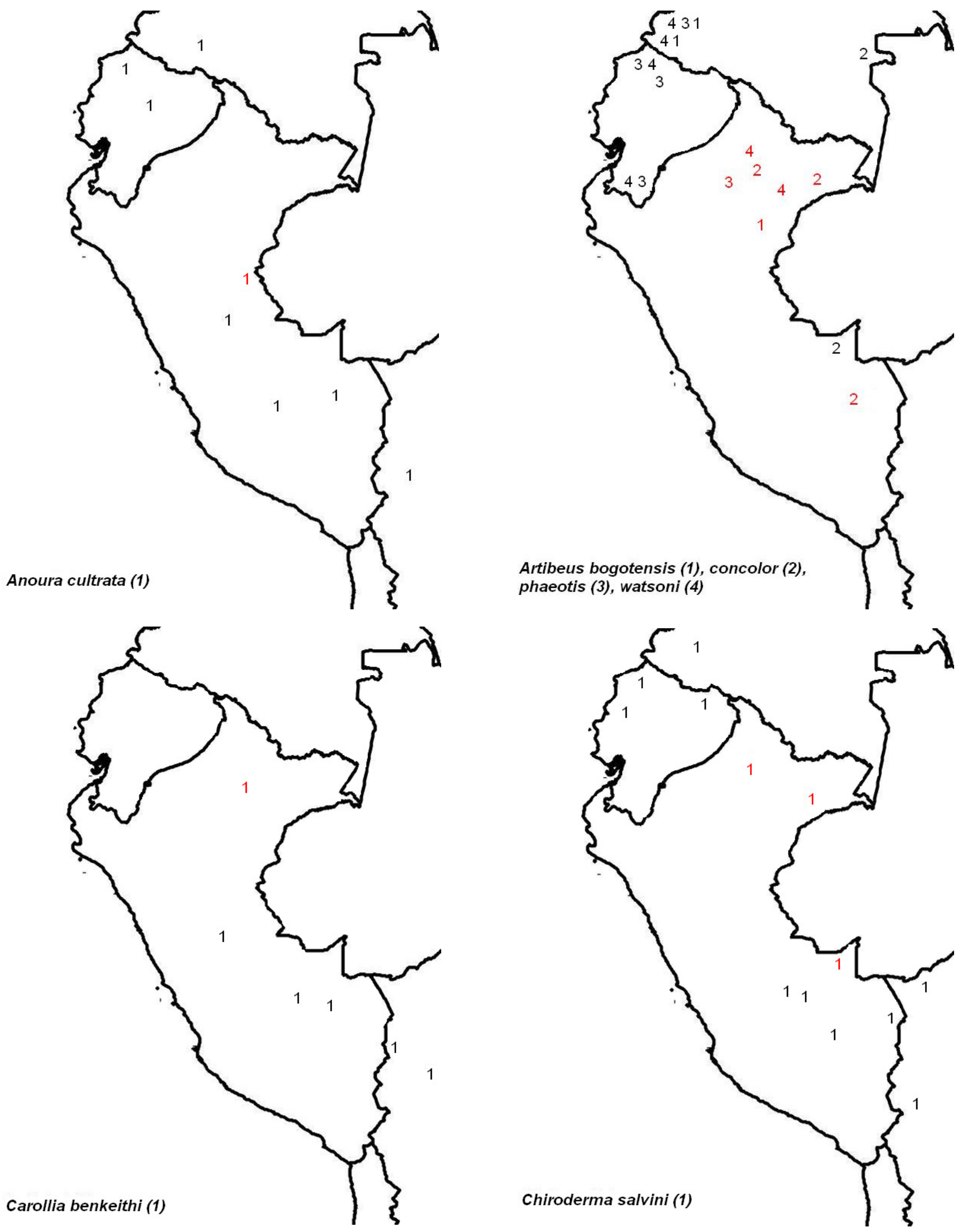

Fig. 2 Especies que extendieron su rango de distribución. En negro localidades previamente conocidas y en rojo las nuevas localidades reportadas desde Gadner (2007). Departamentos de Loreto, Ucayali y Madre de Dios con sus respectivos Anoura cultrata: registros previos (1 en negro) y nueva extensión (1 en rojo, Reserva Nacional Sierra del Divisor - SD); Artibeus bogotensis: registros previos (1 en negro) y nueva extensión (1 en rojo, Centro de Investigaciones Jenaro Herrera - JH); Artibeus concolor: registros previos (2 en negro) y nueva extensión (2 en rojo, Estación Biológica Madre Selva - MS, Carretera Iquitos-Nauta - IN, Los Amigos Conservation Concession - LA); Artibeus phaeotis: registros previos (3 en negro) y nueva extensión (3 en rojo, Alto Río Itaya - RI); Artibeus watsoni: registros previos (4 en negro) y nueva extensión (4 en rojo, Estación Biológica Quebrada Blanco - QB, Reserva Forestal Santa Cruz - SC); Carollia benkeithi: registros previos (1 en negro) y nuevos registros (1 en rojo, Carretera Iquitos-Nauta - IN); Chiroderma salvini: registros previos (1 en negro) y nuevos registros ( 1 en rojo, Estación Biológica Madre Selva - MS, Río Curanja - RC, Reserva Forestal Santa Cruz - SC). 

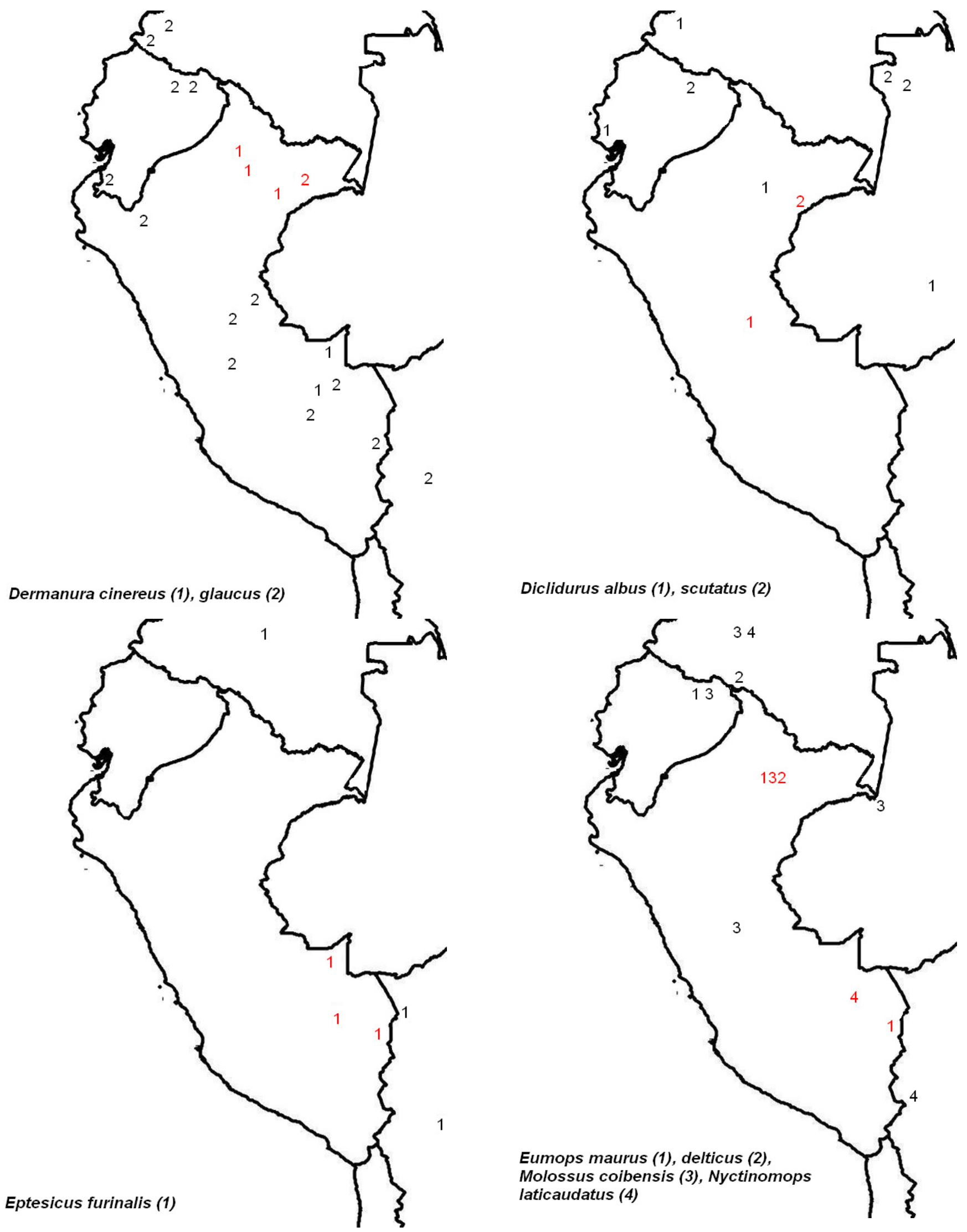

Dermanura cinereus: registros previos (1 en negro) y nuevos registros (1 en rojo, Carretera Iquitos-Nauta - IN, Estación Biológica Quebrada Blanco - QB, Reserva Forestal Santa Cruz - SC); Dermanura glaucus: registros previos (2 en negro) y nuevos registros (2 en rojo, Estación Biológica Madre Selva - MS); Diclidurus albus: registros previos (1 en negro) y nuevos registros (1 en rojo, Pucallpa - PU); Diclidurus scutatus: registros previos (2 en negro) y nuevos registros (2 en rojo, Lago Preto Conservation Concession - LP); Eptesicus furinalis: registros previos (1 en negro) y nuevos registros (1 en rojo, Río Curanja - RC, Morro Leguía - ML, Santuario Nacional Pampas del Heath - PH); Eumops maurus: registros previos (1 en negro) y nuevos registros ( 1 en rojo, Carretera Iquitos-Nauta - IN, Santuario Nacional Pampas del Heath - PH); Eumops delticus: registros previos ( 2 en negro) y nuevos registros ( 2 en rojo, Carretera Iquitos-Nauta - IN); Molossus coibensis: registros previos (3 en negro) y nuevos registros (3 en rojo, Carretera Iquitos-Nauta - IN); Nyctinomops laticaudatus: registros previos (4 en negro) y nuevos registros (4 en rojo, Pakitza - PK); 

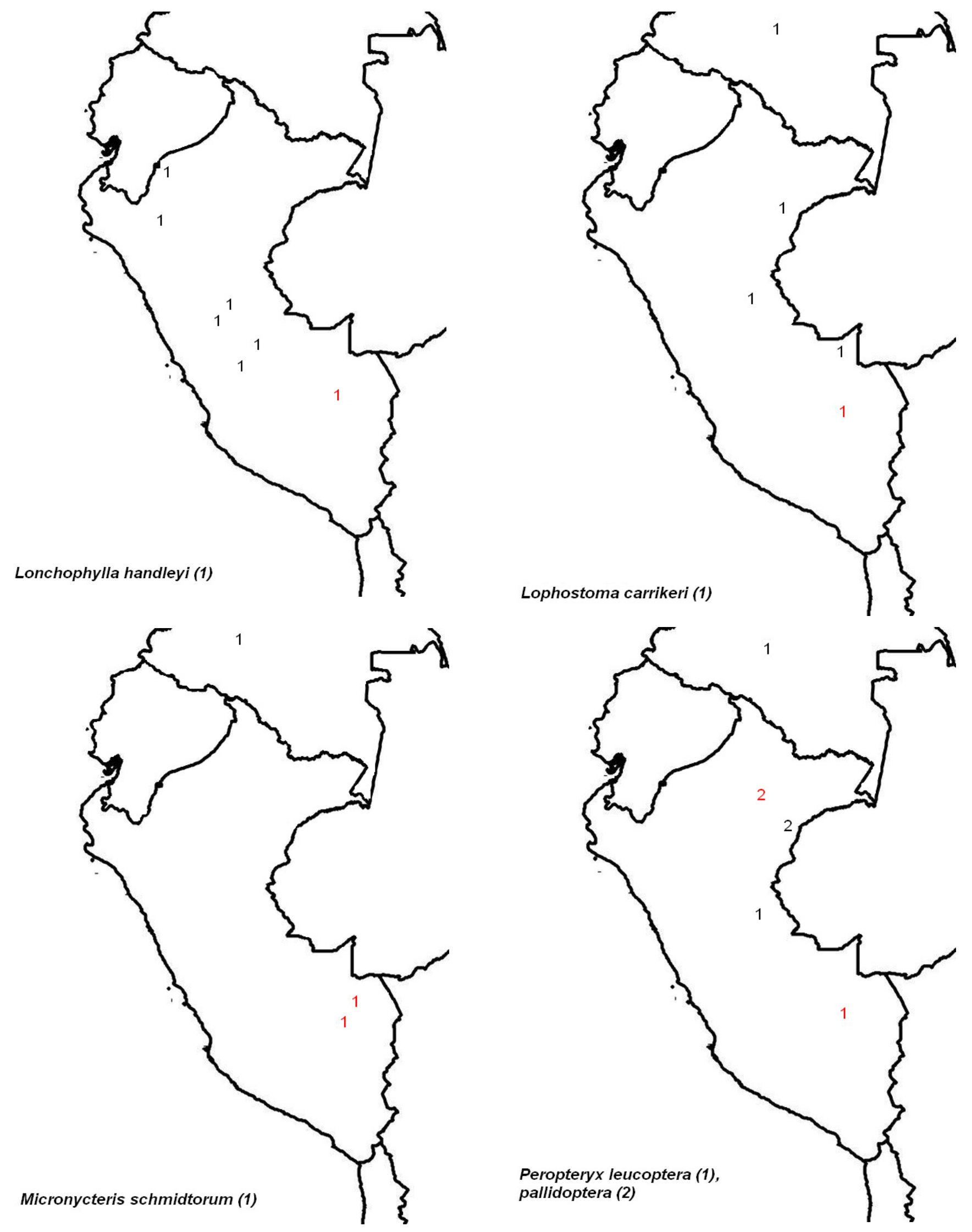

Lonchophylla handleyi: registros previos (1 en negro) y nuevos registros (1 en rojo, Aguas Calientes - AC); Lophostoma carrikeri: registros previos (1 en negro) y nuevos registros (1 en rojo, Maskoitania-MK); Micronycteris schmidtorum: registros previos (1 en negro) y nuevos registros (1 en rojo, Hacienda Amazonia - HA, Pakitza - PK); Peropteryx leucoptera: registros previos (1 en negro) y nuevos registros (1 en rojo, Aguas Calientes - AC); Peropteryx pallidiotera: registros previos (2 en negro) y nuevos registros ( 2 en rojo, Carretera Iquitos-Nauta - IN); 


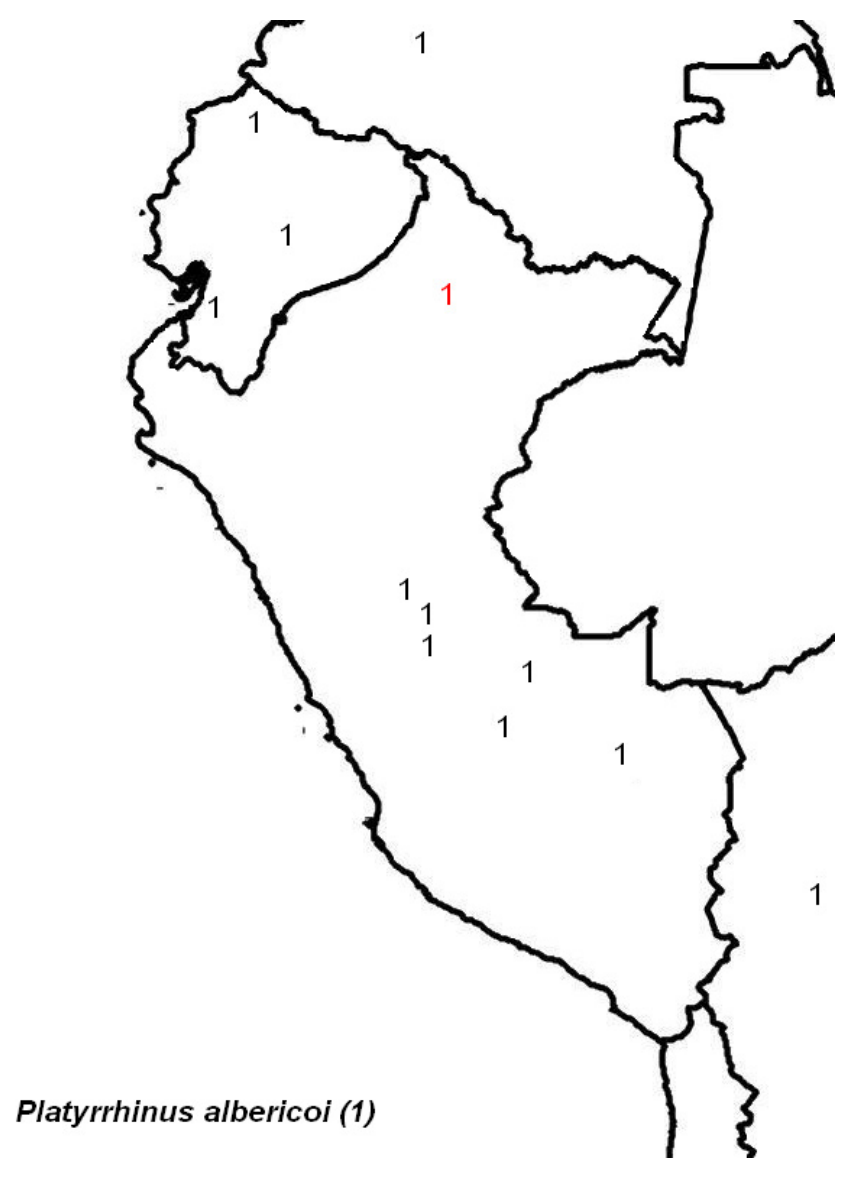

Platyrrhinus albericoi: registros previos (1 en negro) y nuevos registros (1 en rojo, Reserva Forestal Santa Cruz - SC). En la presente Figura 2 se presentan los registros previos (números en color negro) y la extensión del rango de distribución (números en color rojo) de 18 especies de quirópteros. También se muestran los primeros registros y la extensión de rango de las 6 nuevas especies para el Perú. Se especifica el lugar del registro que a su vez actualiza a la referencia básica (Gardner 2007).

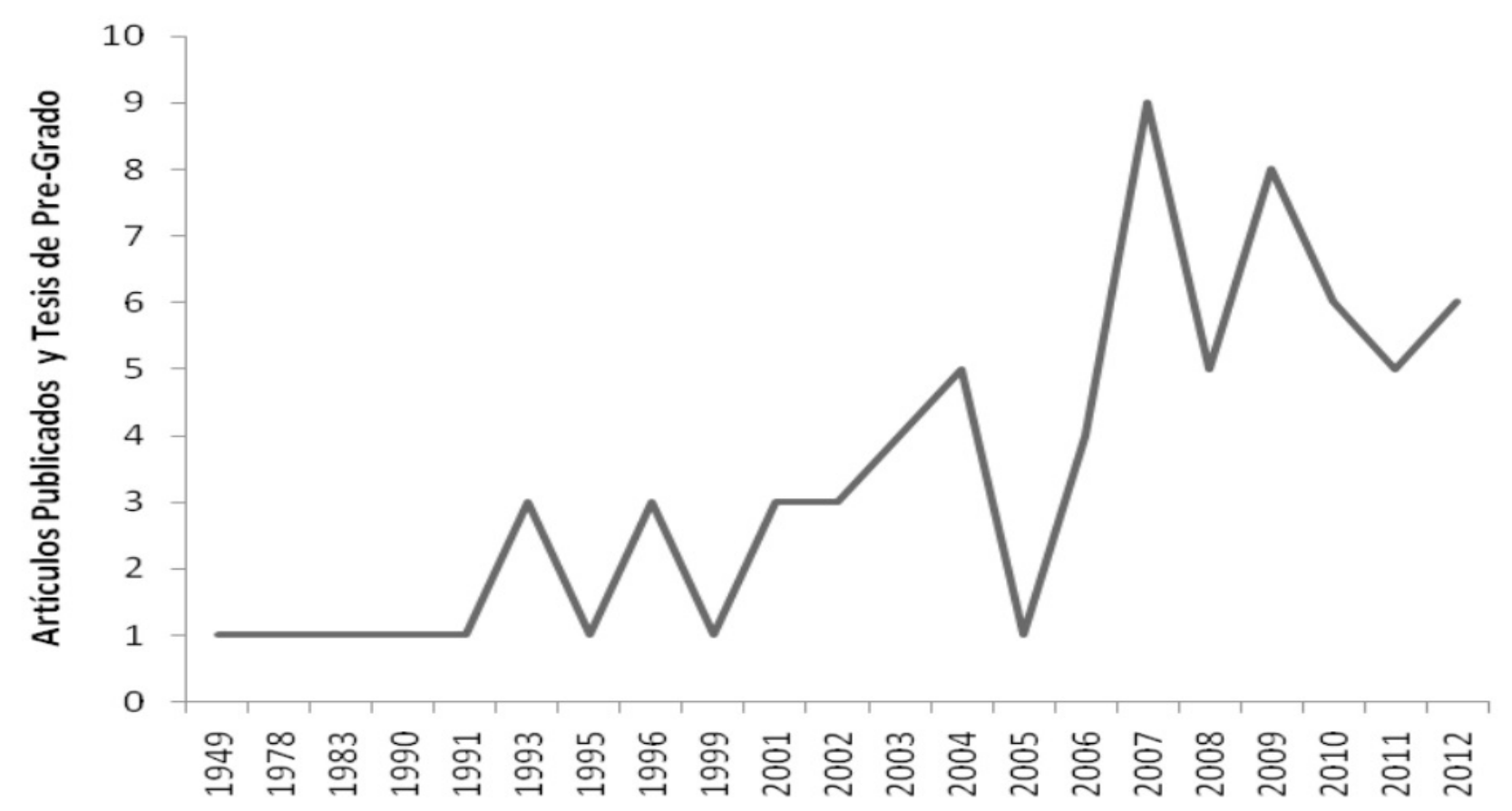

Fig. 3 Artículos publicados y tesis de pre-grado 
rango de distribución de otras 18 especies (Figura 2). El total de especies registradas se distribuye entre las familias Phyllostomidae $(63,8 \%, 81$ especies y 37 géneros), Molossidae (13,4\%, 17 especies y 7 géneros), Emballonuridae (8,7\%, 11 especies y 6 géneros), Vespertilionidae $(8,7 \%$, 11 especies y 3 géneros), Thyropteridae $(2,4 \%$, 3 especies y 1 género), Noctilionidae $(1,6 \%, 2$ especies y 1 género), Furipteridae $(0,7 \%, 1$ especie y 1 género), y Mormoopidae $(0,7 \%, 1$ especie y 1 género) (Tabla 1).

Las 18 especies (Anexo 2) que mostraron un incremento en su rango de distribución son: Anoura cultrata (Quintana \& Pacheco 2009), Artibeus concolor (Angulo 2006, Klingbeil 2007, Arias 2008, Bravo et al. 2008), Carollia benkeithi (Klingbeil 2007, Arias 2008), Chiroderma salvini (Angulo 2006, Quintana \& Pacheco 2009, Saavedra 2010), Dermanura cinereus (Saavedra 2010), Dermanura glaucus (Angulo 2006), Diclidurus albus (Quintana \& Pacheco 2009), Diclidurus scutatus (Escobedo 2012), Eptesicus furinalis (Solari et al. 2006, Pacheco 2009, Quintana \& Pacheco 2009), Eumops maurus (Pacheco 2009, Díaz 2011), Molossus coibensis (Díaz 2011), Nyctinomops laticaudatus, Lonchophylla handleyi, Lophostoma carrikeri, Micronycteris schmidtorum, Peropteryx leucoptera (Solari et al. 2006), Peropteryx pallidoptera (Díaz 2011), y Platyrrhinus albericoi (Saavedra 2010).

En el presente trabajo se reportan 4 especies consideradas por la IUCN (2012) como "Casi Amenazadas/Near threatened" (Anoura cultrata, Sturnira oporaphilum, Vampyrum spectrum), y "Vulnerables/Vulnerables" (Vampyressa melissa). Sin embargo, estas especies no son incluidas en las listas oficiales de especies amenazadas que fueron publicadas por el Ministerio de Agricultura del Perú $(1999,2004)$.

\section{Estudios sobre Quirópteros en Perú}

Se reporta un incremento en la publicación de artículos (revistas indexadas y no indexadas) y en la realización de tesis de pre-grado (Universidad Nacional de la Amazonia Peruana) orientados hacia el estudio de diversos aspectos de la historia natural del grupo de los quirópteros del Perú (Figura 2). La presente figura implicó la revisión de 72 fuentes bibliograficas, los mismos que realizaron algún tipo de estudio ecológico, inventario u otro tema. Estos estudios abarcaron fechas de publicacion que van desde el año 1949 para el más antiguo
(Sanborn 1949) y hasta el año 2012 para el más reciente (Calderón \& Pacheco 2012).

\section{DiscuSIÓN}

Los “artículos de revisión" son de suma importancia hoy en día, debido al incremento del número de publicaciones científicas. Las revisiones pueden ser una solución para mantenerse actualizados acerca de los últimos conocimientos y tendencias sobre un determinado campo de estudio. Sin embargo, muchas revisiones pueden ser subjetivas, dudosas (Cué et al. 1996), e incluso no alcanzan a incluir artículos publicados en revistas no indexadas ni trabajos de tesis de pre-grado de universidades públicas. El presente trabajo pretendió realizar una revisión actualizada de las especies de quirópteros reportadas para los Departamentos de la selva baja del Perú (Loreto, Ucayali y Madre de Dios), valiéndose de varias fuentes bibliográficas.

La elevada proporción de especies de la familia Phyllostomidae frente al resto de familias podría ser el resultado de su rápida adaptación y diversificación (Brosset \& Dominique 1990), para ocupar los diferentes nichos disponibles en el ambiente. Las especies de esta familia suelen ser capturadas utilizando redes de niebla (Fenton et al. 1992, Kalko et al. 1996, Voss \& Emmons 1996), que normalmente cubren el estrato inferior hasta los 3 metros desde el nivel del suelo. Sin embargo, el uso de redes de niebla implicaría un sesgo metodológico importante que podría favorecer la captura de especies de esta familia. Al contrario, los murciélagos insectívoros de las familias Emballonuridae, Vespertilionidae (Fleming 1986, Sánchez-Palomino \& Rivas-Pava 1993, Kalko et al. 1996, Bernard 2002, Bernard \& Fenton 2002), y Molossidae (Fleming 1986, Kalko et al. 1996, Bernard \& Fenton 2002) suelen ser difíciles de capturar debido a su vuelo a mayor altura y por su desarrollado sistema de ecolocación que les permite detectar y evitar las redes de niebla (Fleming 1986, Sánchez-Palomino \& Rivas-Pava 1993, Kalko et al. 1996, Wilson et al. 1996, Simmons \& Voss 1998, Bernard 2002). Un sesgo metodológico al reportar diferencias en las tasas de capturas entre las familias podría implicar la posibilidad de sobreestimar y/o subestimar la presencia de las especies. Al respecto, se sugiere el uso combinado de diferentes técnicas tales como las redes de niebla, trampas harpa, búsquedas en refugios y monitoreos acústicos, para una evaluación más completa de los quirópteros neotropicales (Kalko 
et al. 1996, Simmons \& Voss 1998, Flaquer et al. 2007). Sin embargo, el uso combinado de estas técnicas es aún incipiente en las regiones tropicales, por lo que todavía existe una evidente falta de información para muchas especies.

La perturbación y destrucción del hábitat es la principal amenaza actual de las especies (Pacheco 2002). Actividades económicas tales como, la extracción de madera y petróleo así como la agricultura y ganadería favorecen la destrucción y modificación de hábitats críticos para la supervivencia de las especies en la Amazonia peruana (Escobedo 2003, Jorge \& Velazco 2006, Bravo \& Borman 2008, Díaz 2011). Así mismo, los vacios de información junto a la evidente falta de manejo, control y fiscalización del uso de recursos naturales dentro de las áreas naturales protegidas y concesiones forestales impiden un conocimiento real del estado de conservación de las especies animales y vegetales (Quintana \& Pacheco 2009). La presente revisión, sin embargo, sugiere que en la última década se observa un fuerte incremento en el número de estudios realizados que abordan diferentes aspectos de la historia natural del grupo de los quirópteros.

Es necesario que los nuevos estudios intenten contribuir a suplir los vacios de información (la zona oeste y sur del Departamento de Loreto, y toda la zona este y oeste de los Departamentos de Ucayali y Madre de Dios), y sugerir recomendaciones que resulten de suma importancia para quienes toman las decisiones respecto a las políticas de manejo y conservación de la vida silvestre.

\section{Agradecimientos}

Agradecer a M. I. Torres, por su invalorable apoyo y dedicación para obtener las copias digitales de las tesis que forman parte del acervo de la biblioteca de la Facultad de Ciencias Biológicas de la Universidad Nacional de la Amazonia Peruana (UNAP). Agradecer a S. M. Saavedra y M. C. Ramos por facilitarme una copia digital de sus respectivas tesis de pre-grado (UNAP). También extender mi agradecimiento para Adrià LópezBaucells, por sus comentarios y sugerencias para con este manuscrito.

\section{REFERENCIAS}

Aguirre, L., Mantilla, H., Miller, B. \& Dávalos, L. 2008. Vampyrum spectrum. In: IUCN 2012. IUCN Red List of Threatened Species. Version 2012.2. <www.iucnredlist.org $>$.

Alberico,M., Cadena,A.,Hernández-Camacho, J. \& MuÑoz-SABA, Y. 2000. Mamíferos (Synapsida: Theria) de Colombia. Biota Colombiana 1: 43-75.

Angulo, S.R. 2003. Hábitos alimenticios de Quirópteros en tres tipos de formaciones vegetales de la carretera Iquitos - Nauta. Práctica de pre-grado I, Univerisdad Nacional de la Amazonia Peruana, Iquitos - Perú.

Angulo, S. R. 2004. Distribución vertical de Quirópteros en dos estratos de bosques en la Estación Biológica Madre Selva, río Orosa, Loreto - Perú. Práctica de pre-grado II, Univerisdad Nacional de la Amazonia Peruana, Iquitos - Perú.

Angulo, S. R. \& DíAz, M. M. 2004. Nuevos registros de Sphaeronycteris toxophyllum para la cuenca amazónica del Perú. Mastozoología Neotropical 11: 233-236.

Angulo, S. R. 2006. Distribución vertical de la comunidad de murciélagos (Mammalia: Chiroptera) en bosque primario de tierra firme, Loreto - Perú. Tesis para obtener el título de Biólogo, Universidad Nacional de la Amazonia Peruana, Iquitos - Perú.

ARIAS, L. C. 2008. Ecomorphological structure of an Amazonian Phyllostomid bat assemblage. Master thesis, Texas Tech University.

Ascorra, C. F., Wilson, D. E. \& Romo, M. 1991. Lista anotada de los Quirópteros del Parque Nacional Manu, Perú. Publ. Mus. Hist. Nat., UNMSM, (A) 42: 1-14.

Ascorra, C. F., Gorchov, D. L. \& Cornejo, F. 1993. The bats from Jenaro Herrera, Loreto, Peru. Mammalia 57: 533-552. DOI: https:// dx.doi.org/10.1515/mamm.1993.57.4.533

Ascorra, C. F., Solari, S. \& Wilson, D. E. 1996. Diversidad y ecología de los quirópteros en Pakitza. In Manu: the Biodiversity of Southeastern Peru. Edited by D.E. Wilson and A. Sandoval. Editora Horizonte, Lima, Peru. pp. 593-612.

BÁrquez, R. \& DíAz, M. 2008. Sturnira oporaphilum. In: IUCN 2012. IUCN Red List of Threatened Species. Version 2012.2. $<$ www.iucnredlist.org 
BERNARD, E. 2002. Diet, activity and reproduction of bat species (Mammalia, Chiroptera) in Central Amazonia. Brazil. Rev. Bras. Zool. 19, 173-188. DOI: http://dx.doi. org/10.1590/S0101-81752002000100016

Bernard, E. \& Fenton, M. B. 2002. Species diversity of bats (Mammalia: Chiroptera) in forest fragments, primary forests, and savannas in central Amazonia, Brazil. Can. J. Zool. 80: 1124-1140. DOI: https:// dx.doi.org/10.1139/z02-094

Bravo, A. \& Borman, R. 2008. Mamíferos. In Ecuador, Perú: Cuyabeno-Güepi. Rapid Biological Inventories. Edited by W. S. Alverson, C. Vriesendorp, A. del Campo, D. K. Moskovits, D. F. Stotz, M. GarcíaDonayre, and L. A. Borbor. Report No. 20. Chicago, IL: The Field Museum. pp. 105-111.

Bravo, A., Harms, K. E., Stevens, R. D. \& Emmons, L. H. 2008. Collpas: Activity Hotspots for Frugivorous Bats (Phyllostomidae) in the Peruvian Amazon. Biotropica 40: 203-210. DOI: https://dx.doi.org/10.1111/j.17447429.2007.00362.X

Bravo, A., Harms, K. E. \& Emmons, L. H. 2010. Puddles created by geophagous mammals are potential mineral sources for frugivorous bats (Stenodermatinae) in the Peruvian Amazon. Journal of Tropical Ecology 26: 173-184. DOI: https://dx.doi. org/10.1017/S0266467409990472

Brosset, A. \& Charles-Dominique, P. 1990. The bats from French Guiana: a taxonomic, faunistic and ecological approach. Mammalia 54: 509-560. DOI: https:// dx.doi.org/10.1515/mamm.1990.54.4.509

Cué, M., DíAz, G., DíAz, A. G. \& VAldéS, M. C. 1996. El artículo de revisión. Resumed 9: 86-96.

Calderón, W. \& Pacheco, V. 2012. First report of Artibeus bogotensis Andersen, 1906 (Chiroptera: Phyllostomidae) for Peru. Check List 8: 1333-1336.

DíAz, M. M. 2011. New records of bats from the northern region of the Peruvian Amazon. Zoological Research 32: 168-178.

EsCobedo, M. 2003. Murciélagos. In Peru: Yavarí. Rapid Biological Inventories. Edited by N. Pitman, C. Vriesendorp, and D. Moskovits. Report No. 11. Chicago, IL: The Field Museum. pp. 82-84.
EsCOBEDO, M. 2012. First confirmed record for Peru of Diclidurus scutatus Peters, 1869 (Chiroptera: Emballonuridae). Check List 8: 554-556. DOI: https://dx.doi. org/10.1590/S0101-81752006000300042

Fenton, M. B., Acharya, L., Audet, D., Hickey, M. B. C., Merriman, C., Obrist, M. K., Syme, D. M. \& Adkins, B. 1992. Phyllostomid bats (Chiroptera: Phyllostomidae) as indicators of habitat disruption in the Neotropics. Biotropica 24: 440-446.

Flaquer, C., Torre, I. \& Arrizabalaga, A. 2007. Comparision of sampling methods for inventory of bat communities. Journal of Mammalogy 88: 526-533. DOI: http:// dx.doi.org/10.1644/06-MAMM-A135R1.1

Fleming, T. H. 1986. The structure of Neotropical bat communities: a preliminary analysis. Revista Chilena de Historia Natural 59: 135-150.

GARDNER, A. L. 2007. Mammals of South America, Vol. 1. Marsupials, Xenarthrans, Shrews, and Bats. The University of Chicago Press.

Hice, C. L., Velazco, P. L. \& Willig, M. R. 2004. Bats of the Reserva Nacional Allpahuayo-Mishana, northeastern Peru, with notes on community structure. Acta Chiropterologica 6: 319-334.

Jorge, M. L. S. P. \& Velazco, P. M. 2006. Mamíferos. In Perú: Sierra del Divisor. Rapid Biological Inventories. Edited by C. Vriesendorp, T. S. Schulenberg, W. S. Alverson, D. K. Moskovits, and J. I. RojasMoscoso. Report No. 17. Chicago, IL: The Field Museum. pp. 98-106.

Kalko, E.K.V., Handley, C.O. \& Handley, D. 1996. Organization, diversity, and long-term dynamics of a Neotropical bat community. In Long-term studies of vertebrate communities. Edited by M.L. Cody and J.A. Smallwood. Academic Press, San Diego. pp. 503-553.

KLINGBeIL, B. T. 2007. The response of bats to landscape structure in amazonian forest: an analysis at multiple scale. Master thesis, Texas Tech University.

Lim, B. K., Engstrom, M. D., Reid, F. A., Simmons, N. B., Voss, R. S. \& FleckA, D. W. 2010. New Species of Peropteryx (Chiroptera: Emballonuridae) from Western Amazonia with Comments on Phylogenetic Relationships within the Genus. Am Mus Novit., 3686: 1-20. 
LóPEZ-Wong, C. 2001. Uso de hábitat por Quirópteros en la Zona Reservada Allpahuayo-Mishana. Tesis para obtener el título de Biólogo, Universidad Nacional de la Amazonia Peruana, Iquitos - Perú.

Mantilla, H. \& Molinari, J. 2008. Anoura cultrata. In: IUCN 2012. IUCN Red List of Threatened Species. Version 2012.2. <www. iucnredlist.org

Medellín, R. A. \& GaOna, O. 1999. Seed dispersal by bats and birds in forest in disturbed habitats of Chiapas, Mexico. Biotropica 31: $478-485$.

Ministerio DE Agricultura. 1999. Decreto Supremo No. 013-99-AG. El Peruano. pp. 173278-173280.

Ministerio de Agricultura. 2004. Decreto Supremo No. 034-2004-AG. El Peruano. pp. 276853-276855.

Pacheco, V., Patterson, B. D., Patton, J. L., EMmons, L. H., Solari, S. \& Ascorra, C. F. 1993. List of mammal species known to occur in Manu Biosphere Reserve, Peru. Publ. Mus. Hist. Nat., UNMSM, (A) 44: $1-12$.

Pacheco, V. 2002. Mamíferos del Perú. In Diversidad y conservación de los mamíferos neotropicales. Edited by G. Ceballos and J. A. Simonetti. Conabio-UNAM. México, D.F. pp. 503-550.

Pacheco, V., Cadenillas, R., Salas, E., Tello, C. \& Zeballos, H. 2009. Diversidad y endemismo de los mamíferos del Perú. Rev. peru. biol. 16: 5-32.

Paglia, A.P., Fonseca, G.A.B. DA, Rylands, A. B., Herrmann, G.,Aguiar,L.M.S.,Chiarello, A. G., Leite, Y. L. R., Costa, L. P., Siciliano, S., KierulfF, M. C. M., Mendes, S. L., Tavares, V. Da C., Mittemeier, R. A. \& Patton, J. L. 2012. Lista Anotada dos Mamíferos do Brasil. Occasional Papers in Conservation Biology, 6: 1-76.

Quintana, H., Pacheco, V. \& Salas, E. 2009. Diversidad y conservación de los mamíferos de Ucayali, Perú. Ecología Aplicada 8: 91103.

Ramos, M. C. 2009. Evaluación de Quirópteros en bosques de Varillal, carretera Iquitos Nauta, Loreto - Perú. Práctica de pre-grado II, Univerisdad Nacional de la Amazonia Peruana, Iquitos - Perú.
Ramos, M. C. \& Cevillano, S. C. 2011. Nicho alimentario de murciélagos frugívoros en bosque de colina, río Itaya, Loreto - Perú. Tesis para obtener el título de Biólogo, Universidad Nacional de la Amazonia Peruana, Iquitos - Perú.

Reis, N. R. dos., Perachi, A. L., Pedro, W. A. \& LimA, I. P. de. 2007. Morcegos do Brasil. Universidade Estadual de Londrina, Brazil.

SAAVEDRA, S. M. 2007. Aspectos ecológicos de los murciélagos (Mammalia: Chiroptera) en la Estación Biológica Quebrada Blanco, río Tahuayo - Loreto. Práctica de pre-grado II, Univerisdad Nacional de la Amazonia Peruana, Iquitos - Perú.

SaAvedra, S. M. \& Villalobos, R. G. 2010. Dispersión de semillas por murciélagos (Mammalia: Chiroptera) en bosque primario, bosque secundario y sistemas de cultivo en la Reserva Forestal Santa Cruz, río Mazán. Tesis para obtener el título de Biólogo, Universidad Nacional de la Amazonia Peruana, Iquitos - Perú.

Salazar, J., Yates, T. L. \& Zalles, L. M. 2002. Mamíferos de Bolivia. In Diversidad y conservación de los mamíferos neotropicales. Edited by G. Ceballos and J. A. Simonetti. Conabio-UNAM. México, D.F. pp. 65-113.

Sanborn, C. C. 1949. Mammals from the Rio Ucayali, Peru. Journal of Mammalogy 30: 277-288.

Sanchez-Palomino, P., Rivas-Pava, P. \& Cadena, A. 1993. Composición, abundancia y riqueza de espécies de la comunidad de murciélagos de bosques de galería en la serranía de la Macarena. Caldasia 17: 301312.

Simmons, N. B. \& Voss, R. S. 1998. The mammals of Paracou, French Guiana: a Neotropical lowland rainforest fauna. Part I. Bats. Bull. Am. Mus. Nat. Hist. 237: 1-219.

Simmons, N. B., Voss, R. S. \& Fleck, D. W. 2002. A New Amazonian Species of Micronycteris (Chiroptera: Phyllostomidae) with Notes on the Roosting Behavior of Sympatric Congeners. Am Mus Novit., 3358: 1-14.

Solari, S., Pacheco, V. \& Vivar, E. 1999. Nuevos registros distribucionales de murciélagos peruanos. Rev. peru. biol. 6: 152-159.

Solari, S., Pacheco, V., Luna, L., Velazco, P. M. \& Patterson, B. D. 2006. Mammals of the Manu Biosphere Reserve. In Mammals and Birds of the Manu Biosphere Reserve, 
Peru. Edited by B. D. Patterson, D. F. Stotz, and S. Solari. Fieldiana Zoology 110: 1322. DOI: http://dx.doi.org/10.3158/00150754(2006)110[13:MOTMBR]2.0.CO;2

Sosa, V. J., Hernández-Salazar, E., HernándeZConrique, D. \& Castro-Luna, A. A. 2008. Murciélagos. In Agroecosistemas cafetaleros de Veracruz: biodiversidad, manejo y conservación. Edited by R. H. Manson, V. Hernández-Ortíz, S. Gallina, and K. Mehltreter. Instituto de Ecología A.C. (INECON) e Instituto Nacional de Ecología (INE-SEMAR-NAT), México, pp. 181-192.

Tavarés, V., Velazco, P. \& Aguirre, L. 2008. Vampyressa melissa. In: IUCN 2012. IUCN Red List of Threatened Species. Version 2012.2. <www.iucnredlist.org

Terrones, C. A. 2003. Quirópteros frugívoros como diseminadores de semillas en el Jardín Botánico "El Huayo" - CIEFOR, Puerto Almendras, Loreto - Perú. Práctica de pre-grado I, Univerisdad Nacional de la Amazonia Peruana, Iquitos - Perú.
Tirira, D. G. 2007. Guía de campo de los mamíferos del Ecuador. Ediciones Murciélago Blanco. Publicación especial sobre los mamíferos del Ecuador 7. Quito.

Voss, R.S. \& Emmons, L. H. 1996. Mammalian diversity in Neotropical lowland rainforests: a preliminary assessment. Bull. Am. Mus. Nat. Hist. 230: 1-115.

Wilson, D. E., Ascorra, C. F. \& Solari, S. 1996. Bats as Indicators of Habitat Disturbance. In Manu: the Biodiversity of Southeastern Peru. Edited by D.E. Wilson and A. Sandoval. Editora Horizonte, Lima, Peru. pp. 613-625.

WiLSON, D. E. 2002. Murciélagos: respuestas al vuelo. Universidad Veracruzana. Smithsonian Institution. Xalapa, México. 
Anexo 1. Lista de especies reportadas para los Departamentos de Loreto, Ucayali y Madre de Dios

\begin{tabular}{|c|c|c|c|}
\hline Familia-Especies & Loreto & Ucayali & Madre de Dios \\
\hline \multicolumn{4}{|l|}{ Emballonuridae } \\
\hline Centronycteris maximiliani & AM, IN & & \\
\hline Cormura brevirostris & $\mathrm{AM}, \mathrm{IN}$ & & HA \\
\hline Diclidurus albus & PR & PU & \\
\hline Diclidurus scutatus & LP & & \\
\hline Peropteryx kappleri & $\mathrm{JH}$ & $?$ & $\mathrm{PK}, \mathrm{VC}$ \\
\hline Peropteryx leucoptera & AM, IN, SI & TU & $\mathrm{AC}$ \\
\hline Peropteryx macrotis & IN, SC & YA & \\
\hline Peropteryx pallidoptera & IN, SJ & & \\
\hline Rhynchonycteris naso & AM, JH, SC, SI, YN & LG, YA & $\mathrm{CC}, \mathrm{PK}$ \\
\hline Saccopteryx bilineata & AM, IM, IN, JH, SI, YN & LG, YA & $\mathrm{MK}, \mathrm{P}, \mathrm{PK}$ \\
\hline Saccopteryx leptura & AM, IN, JH, MS, QB, SC, SI, YN & YA & $\mathrm{AC}, \mathrm{CC}, \mathrm{P}$ \\
\hline \multicolumn{4}{|l|}{ Furipteridae } \\
\hline Furipterus horrens & JH, QV & $\mathrm{RD}$ & HA, PK, T \\
\hline \multicolumn{4}{|l|}{ Molossidae } \\
\hline Cynomops abrasus & & $\mathrm{PU}, \mathrm{RC}$ & \\
\hline Cynomops paranus & & $\mathrm{PU}, \mathrm{RC}$ & \\
\hline Cynomops planirostris & $\mathrm{IN}$ & & \\
\hline Eumops auripendulus & & $?$ & \\
\hline Eumops delticus & $\mathrm{IN}$ & & \\
\hline Eumops hansae & QB & & \\
\hline Eumops maurus & $\mathrm{IN}$ & & $\mathrm{PH}$ \\
\hline Eumops trumbulli & & YA & \\
\hline Molossops neglectus & $\mathrm{JH}$ & & \\
\hline Molossops temminckii & $\mathrm{AM}$ & & \\
\hline Molossus coibensis & $\mathrm{IN}$ & & \\
\hline Molossus molossus & $\mathrm{AM}, \mathrm{JH}, \mathrm{QB}, \mathrm{SC}$ & YA & HA, HE, PK, SH, T \\
\hline Molossus rufus & $\mathrm{AM}, \mathrm{IN}, \mathrm{JH}$ & LG, YA & $\mathrm{CO}$ \\
\hline Molossus sinaloae & $\mathrm{YN}$ & & \\
\hline Nyctinomops laticaudatus & & & PK \\
\hline Promops centralis & $\mathrm{AM}, \mathrm{JH}$ & & \\
\hline Tadarida brasiliensis & & & PI \\
\hline
\end{tabular}




\begin{tabular}{|c|c|c|c|}
\hline Familia-Especies & Loreto & Ucayali & Madre de Dios \\
\hline \multicolumn{4}{|l|}{ Mormoopidae } \\
\hline Pteronotus gymnonotus & & YA & \\
\hline \multicolumn{4}{|l|}{ Noctilionidae } \\
\hline Noctilio albiventris & $\mathrm{AM}, \mathrm{IN}, \mathrm{JH}$ & PU, YA & $\mathrm{CC}, \mathrm{MK}, \mathrm{PK}$ \\
\hline Noctilio leporinus & $\mathrm{IN}$ & SR, YA & PK \\
\hline \multicolumn{4}{|l|}{ Phyllostomidae } \\
\hline Anoura caudifer & IN, JH, SI, YN & $\mathrm{RC}$ & $\begin{array}{l}\mathrm{AC}, \mathrm{CO}, \mathrm{CP}, \mathrm{HA}, \mathrm{HE}, \mathrm{MK}, \mathrm{PK}, \\
\text { SP, SU, T }\end{array}$ \\
\hline Anoura cultrata & & $\mathrm{SD}, \mathrm{AD}$ & $\mathrm{CP}, \mathrm{SU}$ \\
\hline Anoura geoffroyi & & $\mathrm{RC}$ & $\mathrm{CO}, \mathrm{CP}, \mathrm{HA}, \mathrm{LE}, \mathrm{PI}, \mathrm{SU}, \mathrm{T}, \mathrm{TE}$ \\
\hline Artibeus bogotensis & $\mathrm{JH}$ & & \\
\hline Artibeus concolor & $\mathrm{IN}, \mathrm{MS}, \mathrm{SC}$ & $\mathrm{RC}$ & LA \\
\hline Artibeus lituratus & $\begin{array}{l}\text { AM, IM, IN, JH, MS, QB, RI, } \\
\text { SC, SI, YN }\end{array}$ & $\mathrm{AG}, \mathrm{PU}, \mathrm{SD}, \mathrm{SR}$ & $\begin{array}{l}\mathrm{AC}, \mathrm{CC}, \mathrm{CO}, \mathrm{HA}, \mathrm{HE}, \mathrm{LA}, \mathrm{MK}, \\
\mathrm{PK}, \mathrm{RP}, \mathrm{T}\end{array}$ \\
\hline Artibeus obscurus & $\begin{array}{l}\text { AM, IM, IN, JH, MS, QB, RI, } \\
\text { SC, SI, YN }\end{array}$ & PU, SD & $\begin{array}{l}\mathrm{AC}, \mathrm{CC}, \mathrm{CJ}, \mathrm{CO}, \mathrm{CP}, \mathrm{CS}, \mathrm{HA}, \\
\mathrm{HE}, \mathrm{LA}, \mathrm{MK}, \mathrm{PK}, \mathrm{RP}, \mathrm{SH}, \mathrm{T}\end{array}$ \\
\hline Artibeus phaeotis & RI & & \\
\hline Artibeus planirostris & $\begin{array}{l}\mathrm{AM}, \mathrm{IN}, \mathrm{JH}, \mathrm{MS}, \mathrm{QB}, \mathrm{RI}, \mathrm{SC}, \\
\text { SI, YN }\end{array}$ & PU, SD & $\begin{array}{l}\mathrm{AC}, \mathrm{CC}, \mathrm{CO}, \mathrm{CS}, \mathrm{HA}, \mathrm{HE}, \mathrm{LA}, \\
\mathrm{M}, \mathrm{MK}, \mathrm{PK}, \mathrm{RP}, \mathrm{T}\end{array}$ \\
\hline Artibeus watsoni & $\mathrm{QB}, \mathrm{SC}$ & & \\
\hline Carollia benkeithi & IN & $\mathrm{SD}, \mathrm{SR}$ & $\begin{array}{l}\mathrm{AC}, \mathrm{CC}, \mathrm{CJ}, \mathrm{CO}, \mathrm{CS}, \mathrm{HA}, \mathrm{LA}, \\
\mathrm{M}, \mathrm{MD}, \mathrm{MK}, \mathrm{PK}, \mathrm{RP}, \mathrm{SH}, \mathrm{T}\end{array}$ \\
\hline Carollia brevicauda & $\begin{array}{l}\mathrm{AM}, \mathrm{IM}, \mathrm{IN}, \mathrm{JH}, \mathrm{MS}, \mathrm{QB}, \mathrm{RI} \\
\mathrm{SC}, \mathrm{SI}, \mathrm{YN}\end{array}$ & $\mathrm{AB}, \mathrm{SD}$ & $\begin{array}{l}\text { AC, BN, CC, CJ, CO, CP, CS, } \\
\text { HA, LA, M, MD, MK, P, PK, } \\
\text { QC, RM, RP, SP, T }\end{array}$ \\
\hline Carollia castanea & $\mathrm{AM}, \mathrm{IN}, \mathrm{MS}, \mathrm{QB}, \mathrm{SC}$ & & \\
\hline Carollia manu & & & $\mathrm{BN}, \mathrm{CP}, \mathrm{ML}, \mathrm{SP}$ \\
\hline Carollia perspicillata & $\begin{array}{l}\text { AM, IM, IN, JH, MS, QB, RI, } \\
\text { SC, SI, YN }\end{array}$ & $\mathrm{BU}, \mathrm{PU}, \mathrm{SD}, \mathrm{YA}$ & $\begin{array}{l}\text { AC, BN, CC, CJ, CO, CP, CS, } \\
\text { HA, HE, LA, M, MD, MK, ML, } \\
\text { P, PK, RP, SH, T }\end{array}$ \\
\hline Chiroderma salvini & $\mathrm{IN}, \mathrm{MS}, \mathrm{SC}$ & $\mathrm{RC}$ & $\mathrm{AC}, \mathrm{CO}, \mathrm{HA}, \mathrm{LA}, \mathrm{MK}, \mathrm{SU}, \mathrm{T}$ \\
\hline Chiroderma trinitatum & $\mathrm{AM}, \mathrm{IN}, \mathrm{JH}, \mathrm{SI}$ & PU, SD & $\begin{array}{l}\mathrm{AC}, \mathrm{CC}, \mathrm{CO}, \mathrm{HA}, \mathrm{LA}, \mathrm{MK}, \mathrm{PK}, \\
\mathrm{T}\end{array}$ \\
\hline Chiroderma villosum & AM, IN, JH, MS, RI, SI, YN & & $\begin{array}{l}\text { AC, CC, CS, HA, LA, MK, PK, } \\
\text { T }\end{array}$ \\
\hline Choeroniscus minor & AM, IN, JH, MS, SC, SI, YN & $\mathrm{RC}$ & $\mathrm{CC}, \mathrm{HA}, \mathrm{MK}, \mathrm{PK}$ \\
\hline Chrotopterus auritus & AM, IN, JH, MS, QB, SC, SI & & $\mathrm{AC}, \mathrm{CC}, \mathrm{HA}, \mathrm{LA}$ \\
\hline Dermanura anderseni & $\begin{array}{l}\text { AM, IM, IN, JH, MS, RI, SC, } \\
\text { SI, YN }\end{array}$ & $\mathrm{RC}$ & $\begin{array}{l}\mathrm{AC}, \mathrm{CC}, \mathrm{CO}, \mathrm{CP}, \mathrm{HA}, \mathrm{HE}, \mathrm{LA}, \\
\mathrm{MD}, \mathrm{MK}, \mathrm{PK}, \mathrm{RP}, \mathrm{SH}\end{array}$ \\
\hline Dermanura cinereus & $\mathrm{IN}, \mathrm{QB}, \mathrm{SC}$ & $\mathrm{RC}$ & LA, PK \\
\hline Dermanura glaucus & $\mathrm{AM}, \mathrm{IN}, \mathrm{MS}, \mathrm{QB}, \mathrm{RI}, \mathrm{SC}$ & SR & $\begin{array}{l}\text { AC, BN, CC, CO, CP, HA, LA, } \\
\text { MK, ML, PI, QC, SP, SU, T, TE }\end{array}$ \\
\hline Dermanura gnomus & $\mathrm{AM}, \mathrm{IN}, \mathrm{JH}, \mathrm{MS}, \mathrm{QB}, \mathrm{SC}$ & PU, SR & $\mathrm{AC}, \mathrm{HA}, \mathrm{MK}, \mathrm{PK}$ \\
\hline Desmodus rotundus & AM, IM, IN, JH, MS, SC, SI & $\mathrm{BU}, \mathrm{PU}, \mathrm{YA}$ & HA, HE, LA, MK, PK \\
\hline Diaemus youngi & $\mathrm{PB}$ & $\mathrm{CL}, \mathrm{YA}$ & \\
\hline Diphylla ecaudata & & $\mathrm{SD}, \mathrm{YA}$ & $\mathrm{CP}, \mathrm{HE}, \mathrm{LA}, \mathrm{PK}$ \\
\hline Enchisthenes hartii & $\mathrm{IN}, \mathrm{JH}$ & $?$ & $\begin{array}{l}\text { AC, BN, CO, CP, HA, LA, MK, } \\
\text { PI, QC, SP, SU, T }\end{array}$ \\
\hline Glossophaga commissarisi & $\mathrm{IM}, \mathrm{IN}, \mathrm{MS}, \mathrm{YN}$ & $\mathrm{PU}$ & $\mathrm{PK}$ \\
\hline
\end{tabular}




\begin{tabular}{|c|c|c|c|}
\hline Familia-Especies & Loreto & Ucayali & Madre de Dios \\
\hline Glossophaga soricina & AM, IM, IN, JH, MS, QB, SC, YN & PU & $\mathrm{CC}, \mathrm{HA}, \mathrm{HE}, \mathrm{LA}, \mathrm{PK}$ \\
\hline Glyphonycteris daviesi & IN & & LA, PK \\
\hline Glyphonycteris sylvestris & IN & & \\
\hline Lampronycteris brachyotis & $\mathrm{JH}, \mathrm{YN}$ & & LA, CJ \\
\hline Lichonycteris degener & & YA & PK \\
\hline Lionycteris spurrelli & $\mathrm{IN}$ & & $\mathrm{AC}, \mathrm{CP}, \mathrm{HA}, \mathrm{MK}$ \\
\hline Lonchophylla handleyi & & $\mathrm{AD}$ & $\mathrm{AC}$ \\
\hline Lonchophylla robusta & & $\mathrm{AD}$ & \\
\hline Lonchophylla thomasi & AM, IN, JH, MS, QB, SC, YN & $\mathrm{RC}$ & $\begin{array}{l}\mathrm{AC}, \mathrm{CC}, \mathrm{CO}, \mathrm{HA}, \mathrm{HE}, \mathrm{LA}, \\
\mathrm{MK}, \mathrm{P}, \mathrm{PK}, \mathrm{RP}\end{array}$ \\
\hline Lonchorhina aurita & & & $\mathrm{CO}, \mathrm{MK}$ \\
\hline Lophostoma brasiliense & AM, IN, JH & $\mathrm{RC}$ & HE, PK, RP \\
\hline Lophostoma carrikeri & $\mathrm{IM}, \mathrm{IN}, \mathrm{JH}$ & $\mathrm{RC}$ & MK \\
\hline Lophostoma silvicolum & AM, IN, JH, MS, QB, SC, SI, YN & $\begin{array}{l}\mathrm{LG}, \mathrm{PU} \\
\mathrm{SD}, \mathrm{YA}\end{array}$ & $\begin{array}{l}\mathrm{AC}, \mathrm{CC}, \mathrm{CP}, \mathrm{CO}, \mathrm{HA}, \mathrm{HE}, \\
\mathrm{IT}, \mathrm{LA}, \mathrm{M}, \mathrm{PK}, \mathrm{RM}\end{array}$ \\
\hline Macrophyllum macrophyllum & & YA & PK \\
\hline Mesophylla macconnelli & AM, IN, JH, MS, QB, RI, SC & SR & $\begin{array}{l}\mathrm{AC}, \mathrm{AL}, \mathrm{CC}, \mathrm{CO}, \mathrm{HA}, \mathrm{LA}, \\
\mathrm{PK}, \mathrm{T}\end{array}$ \\
\hline Micronycteris hirsuta & & & $\mathrm{AC}, \mathrm{RP}$ \\
\hline Micronycteris matses & SJ & & \\
\hline Micronycteris megalotis & $\mathrm{AM}, \mathrm{IN}, \mathrm{JH}, \mathrm{QB}, \mathrm{SI}$ & $\mathrm{AC}, \mathrm{YA}$ & $\begin{array}{l}\mathrm{CO}, \mathrm{HA}, \mathrm{LA}, \mathrm{MK}, \mathrm{PI}, \mathrm{PK}, \\
\mathrm{RP}\end{array}$ \\
\hline Micronycteris minuta & AM, IM, IN, QB, SC & & HA, LA, MK, PK \\
\hline Micronycteris schmidtorum & & & HA, PK, RP \\
\hline Mimon crenulatum & AM, IN, JH, MS, QB, SC, SI, YN & YA & $\mathrm{CO}, \mathrm{HA}, \mathrm{LA}, \mathrm{PK}$ \\
\hline Phylloderma stenops & AM, IN, JH, MS, SC & SD & $\mathrm{AC}, \mathrm{CC}, \mathrm{CO}, \mathrm{LA}, \mathrm{PK}$ \\
\hline Phyllostomus discolor & AM, IM, IN, JH, MS, SC & $?$ & \\
\hline Phyllostomus elongatus & AM, IM, IN, JH, MS, QB, SC, SI, YN & SR & $\begin{array}{l}\mathrm{AC}, \mathrm{CC}, \mathrm{CO}, \mathrm{CS}, \mathrm{HA}, \mathrm{HE}, \\
\mathrm{LA}, \mathrm{M}, \mathrm{MK}, \mathrm{PK}, \mathrm{QC}, \mathrm{T}\end{array}$ \\
\hline Phyllostomus hastatus & AM, IM, IN, JH, MS, QB, SC, SI, YN & $\begin{array}{l}\mathrm{AC}, \mathrm{SD} \\
\mathrm{SR}, \mathrm{YA}\end{array}$ & $\begin{array}{l}\mathrm{AC}, \mathrm{BV}, \mathrm{CC}, \mathrm{CO}, \mathrm{HA}, \mathrm{HE}, \\
\mathrm{IT}, \mathrm{LA}, \mathrm{MK}, \mathrm{PK}, \mathrm{RP}\end{array}$ \\
\hline Platyrrhinus albericoi & $\mathrm{SC}$ & $?$ & PI, SP, SU \\
\hline Platyrrhinus brachycephalus & AM, IM, IN, JH, MS, QB & PU, SD & $\begin{array}{l}\mathrm{AC}, \mathrm{CC}, \mathrm{CO}, \mathrm{HA}, \mathrm{HE}, \mathrm{LA}, \\
\mathrm{MK}, \mathrm{PK}, \mathrm{RP}, \mathrm{T}\end{array}$ \\
\hline Platyrrhinus helleri & AM, IN, JH, MS, SI, YN & PU & $\begin{array}{l}\mathrm{AC}, \mathrm{CC}, \mathrm{CO}, \mathrm{HA}, \mathrm{HE}, \mathrm{LA}, \\
\mathrm{MK}, \mathrm{PK}, \mathrm{RP}, \mathrm{T}\end{array}$ \\
\hline Platyrrhinus incarum & & $\mathrm{SD}, \mathrm{YA}$ & \\
\hline Platyrrhinus infuscus & AM, IN, SC & $\mathrm{RC}, \mathrm{SD}$ & $\begin{array}{l}\mathrm{AC}, \mathrm{CO}, \mathrm{CP}, \mathrm{HA}, \mathrm{HE}, \mathrm{IT}, \\
\mathrm{LA}, \mathrm{MK}, \mathrm{PK}, \mathrm{RP}, \mathrm{T}\end{array}$ \\
\hline Platyrrhinus masu & & & $\begin{array}{l}\text { CO, CP, HA, HE, LE, PI, } \\
\text { QC, SP, SU, TE }\end{array}$ \\
\hline Platyrrhinus nigellus & & & $\begin{array}{l}\mathrm{AC}, \mathrm{BN}, \mathrm{CO}, \mathrm{CP}, \mathrm{HA}, \mathrm{QC}, \\
\mathrm{RP}, \mathrm{SP}, \mathrm{T}\end{array}$ \\
\hline Rhinophylla fischerae & AM, IN, JH, MS, QB, SC, SI & SR & \\
\hline Rhinophylla pumilio & AM, IN, JH, MS, QB, SC, SI, YN & $\mathrm{SR}$ & $\mathrm{AC}, \mathrm{HA}, \mathrm{HE}, \mathrm{LA}, \mathrm{MK}, \mathrm{PK}$ \\
\hline Sphaeronycteris toxophyllum & IQ & & $\mathrm{CC}, \mathrm{LA}$ \\
\hline Sturnira erythromos & & $\mathrm{AD}$ & $\begin{array}{l}\mathrm{AA}, \mathrm{BN}, \mathrm{HA}, \mathrm{LE}, \mathrm{ML}, \mathrm{PI} \\
\text { SP, SU, TE }\end{array}$ \\
\hline Sturnira lilium & AM, IN, JH, MS, RI, SC, YN & YA & $\begin{array}{l}\mathrm{AC}, \mathrm{CO}, \mathrm{HA}, \mathrm{HE}, \mathrm{LA}, \mathrm{MK}, \\
\mathrm{PK}, \mathrm{QC}, \mathrm{RP}, \mathrm{SP}, \mathrm{T}\end{array}$ \\
\hline
\end{tabular}




\begin{tabular}{|c|c|c|c|}
\hline Familia-Especies & Loreto & Ucayali & Madre de Dios \\
\hline Sturnira magna & AM, IN, JH, RI, SC, SI & $\mathrm{SD}, \mathrm{AD}$ & AC, CO, CP, HA, HE, SP, SU, T \\
\hline Sturnira oporaphilum & & $?$ & $\mathrm{BN}, \mathrm{CO}, \mathrm{CP}, \mathrm{HA}, \mathrm{SP}, \mathrm{T}$ \\
\hline Sturnira tildae & $\mathrm{AM}, \mathrm{IN}, \mathrm{JH}, \mathrm{MS}, \mathrm{QB}, \mathrm{RI}, \mathrm{SC}$ & $\mathrm{RC}, \mathrm{SD}$ & AC, HA, LA, MK, PK \\
\hline Tonatia saurophila & $\mathrm{AM}, \mathrm{IN}, \mathrm{QB}, \mathrm{SC}$ & YA & $\mathrm{CC}, \mathrm{CP}, \mathrm{HA}, \mathrm{LA}, \mathrm{PK}$ \\
\hline Trachops cirrhosus & AM, IM, IN, JH, MS, QB, SC, SI, YN & $\mathrm{RC}, \mathrm{SD}$ & $\begin{array}{l}\text { AC, CC, CP, CS, HE, LA, M, } \\
\text { MK, PK }\end{array}$ \\
\hline Trinycteris nicefori & $\mathrm{AM}, \mathrm{IN}, \mathrm{JH}, \mathrm{QB}, \mathrm{SC}, \mathrm{SI}, \mathrm{YN}$ & $\mathrm{RC}$ & PK \\
\hline Uroderma bilobatum & $\begin{array}{l}\text { AM, IM, IN, JH, MS, QB, RI, SC, SI, } \\
\text { YN }\end{array}$ & CT, PU & $\begin{array}{l}\text { AC, CC, CO, CP, HA, HE, LA, } \\
\text { MK, PK, RP, T }\end{array}$ \\
\hline Uroderma magnirostrum & AM, IN, JH, MS, RI, YN & $\mathrm{RC}$ & $\mathrm{AC}, \mathrm{CC}, \mathrm{LA}, \mathrm{MK}, \mathrm{PK}$ \\
\hline Vampyressa melissa & & $?$ & $\mathrm{BN}, \mathrm{CO}, \mathrm{QC}, \mathrm{SP}$ \\
\hline Vampyressa thyone & $\mathrm{IN}, \mathrm{MS}, \mathrm{QB}, \mathrm{SC}$ & $\mathrm{RC}, \mathrm{SD}$ & $\mathrm{AC}, \mathrm{CC}, \mathrm{CO}, \mathrm{HA}, \mathrm{PK}, \mathrm{T}$ \\
\hline Vampyriscus bidens & $\mathrm{AM}, \mathrm{IN}, \mathrm{MS}, \mathrm{QB}, \mathrm{RI}, \mathrm{SC}$ & $\mathrm{BU}$ & AC, CP, CS, HA, LA, PK, T \\
\hline Vampyriscus brocki & $\mathrm{AM}, \mathrm{IN}, \mathrm{JH}, \mathrm{MS}, \mathrm{RI}$ & & \\
\hline Vampyrodes caraccioli & $\mathrm{AM}, \mathrm{IN}, \mathrm{QB}, \mathrm{SC}$ & $\mathrm{RC}$ & $\mathrm{AC}, \mathrm{CO}, \mathrm{CP}, \mathrm{HA}, \mathrm{LA}, \mathrm{MK}, \mathrm{PK}$ \\
\hline Vampyrum spectrum & $\mathrm{IN}, \mathrm{JH}, \mathrm{QB}$ & YA & $\mathrm{CC}, \mathrm{HA}$ \\
\hline \multicolumn{4}{|l|}{ Thyropteridae } \\
\hline Thyroptera discifera & $\mathrm{JH}$ & $\mathrm{CM}$ & \\
\hline Thyroptera lavali & IN & $\mathrm{VH}$ & MK \\
\hline Thyroptera tricolor & AM, IM, IN, YN & SR & $\mathrm{CC}, \mathrm{HA}, \mathrm{LA}, \mathrm{PK}$ \\
\hline \multicolumn{4}{|l|}{ Vespertilionidae } \\
\hline Eptesicus brasiliensis & $\mathrm{AM}, \mathrm{IN}, \mathrm{JH}$ & $\mathrm{RC}$ & LE, PI, SU, T \\
\hline Eptesicus chiriquinus & & & $\mathrm{CO}, \mathrm{MK}$ \\
\hline Eptesicus furinalis & & $\mathrm{RC}$ & $\mathrm{ML}, \mathrm{PH}, \mathrm{T}$ \\
\hline Lasiurus blossevillii & & $\mathrm{RC}$ & PI \\
\hline Lasiurus ega & $\mathrm{AM}$ & $\mathrm{RC}$ & PK \\
\hline Myotis albescens & $\mathrm{AM}, \mathrm{IN}, \mathrm{JH}, \mathrm{YN}$ & YA & AC, HE, MK, PK \\
\hline Myotis keaysi & & & AC, HA, MK, PI, SP, SU, TE \\
\hline Myotis nigricans & AM, IN, JH, QB, SC, SI, YN & AG, SR, YA & $\begin{array}{l}\text { AC, CC, CO, HA, HE, MK, PK, } \\
\text { RP, SH, T, VC }\end{array}$ \\
\hline Myotis oxyotus & & & LE, PI \\
\hline Myotis riparius & $\mathrm{AM}, \mathrm{IN}, \mathrm{JH}, \mathrm{MS}, \mathrm{QB}, \mathrm{SC}, \mathrm{YN}$ & ?, SD & $\mathrm{AC}, \mathrm{CO}, \mathrm{HA}, \mathrm{HE}, \mathrm{MK}, \mathrm{PK}, \mathrm{SP}$ \\
\hline Myotis simus & $\mathrm{AM}, \mathrm{IN}, \mathrm{JH}, \mathrm{QB}$ & YA & PK \\
\hline
\end{tabular}

Loreto: AM (Reserva Nacional Allpahuayo-Mishana), IM (Isla Muyuy), IN (Carretera Iquitos-Nauta), IQ (Iquitos), JH (Centro de Investigaciones Jenaro Herrera), LP (Lago Preto Conservation Concession), MS (Estación Biológica Madre Selva), PB (Pebas), PR (Parinari), QB (Estación Biológica Quebrada Blanco), QV (Quebrada Vainilla), RI (Alto Río Itaya), SC (Reserva Forestal Santa Cruz), SI (Sucusari), SJ (Nuevo Sam Juan), YN (Yanamono). Ucayali: AB (Abra Boquerón), AD (Abra Divisoria), AG (Agua Caliente), BU (Boca del Río Urubamba), CL (Colonia Callaria), CM (Cumaria), CT (Contamana), LG (Lagarto), PU (Pucallpa), RC (Río Curanja), RD (Río Disqui), SD (Reserva Nacional Sierra del Divisor), SR (Santa Rosa), TU (Tushemo), VH (Bosque Nacional Alexander von Humboldt), YA (Yarinacocha). Madre de Dios: AA (Abra Acjanaco), AC (Aguas Calientes), AL (Altamira), BN (Albergue Bosque de las Nubes), BV (Puerto Buena Vista), CC (Cocha Cashu), CJ (Cocha Juares), CO (Consuelo), CP (Cerro de Pantiacolla), CS (Cocha Salvador), HA (Hacienda Amazonia), HE (Hacienda Erika), IT (Itahuania), LA (Los Amigos Conservation Concession), LE (La Esperanza), M (Manu), MD (Alto Río Madre de Dios), MK (Maskoitania), ML (Morro Leguía), P (Pilcopata), PH (Santuario Nacional Pampas del Heath), PI (Pillahuata), PK (Pakitza), QC (Quitacalzon), RM (Río Manu), RP (Palotoa), SH (Shintuya), SP (San Pedro), SU (Suecia), T (Tono), TE (Trocha Ericcson), VC (Hacienda Villa Carmen). ?: Especie reportada para el Departamento pero no se precisa el sitio de estudio. La presente Tabla 1 incorpora todos los sitios donde se registraron especies de quirópteros. Los sitios que actualizan a la referencia básica se encuentran también se encuentra en la Figura 1. 